\title{
Contextual Multi-Armed Bandits for Link Adaptation in Cellular Networks
}

\author{
Vidit Saxena \\ vidits@kth.se \\ KTH Royal Institute of Technology \\ Stockholm, Sweden \\ Mats Bengtsson \\ matben@kth.se \\ KTH Royal Institute of Technology \\ Stockholm, Sweden
}

\author{
Joakim Jaldén \\ jalden@kth.se \\ KTH Royal Institute of Technology \\ Stockholm, Sweden \\ Hugo Tullberg \\ hugo.tullberg@ericsson.com \\ Ericsson $\mathrm{AB}$ \\ Stockholm, Sweden
}

\author{
Joseph E. Gonzalez \\ jegonzal@berkeley.edu \\ University of California \\ Berkeley, California \\ Ion Stoica \\ istoica@berkeley.edu \\ University of California \\ Berkeley, California
}

\begin{abstract}
Cellular networks dynamically adjust the transmission parameters for a wireless link in response to its time-varying channel state. This is known as link adaptation, where the typical goal is to maximize the link throughput. State-of-the-art outer loop link adaptation (OLLA) selects the optimal transmission parameters based on an approximate, offline, model of the wireless link. Further, OLLA refines the offline model by dynamically compensating any deviations from the observed link performance. However, in practice, OLLA suffers from slow convergence and a sub-optimal link throughput. In this paper, we propose a link adaptation approach that overcomes the shortcomings of OLLA through a novel learning scheme. Our approach relies on contextual multi-armed bandits (MAB), where the context vector is composed of the instantaneous wireless channel state along with side information about the link. For a given context, our approach learns the success probability for each of the available transmission parameters, which is then exploited to select the throughput-maximizing parameters. Through numerical experiments, we show that our approach converges faster than OLLA and achieves a higher steady-state link throughput. For frequent and infrequent channel reports respectively, our scheme outperforms OLLA by $15 \%$ and $25 \%$ in terms of the steady-state link throughput.
\end{abstract}

\section{CCS CONCEPTS}

- Networks $\rightarrow$ Wireless access points, base stations and infrastructure; Network control algorithms; • Computing methodologies $\rightarrow$ Online learning settings.

\section{KEYWORDS}

cellular networks, outer loop link adaptation, contextual multiarmed bandits, artificial neural networks

Permission to make digital or hard copies of all or part of this work for personal or classroom use is granted without fee provided that copies are not made or distributed for profit or commercial advantage and that copies bear this notice and the full citation on the first page. Copyrights for components of this work owned by others than ACM must be honored. Abstracting with credit is permitted. To copy otherwise, or republish, to post on servers or to redistribute to lists, requires prior specific permission and/or a fee. Request permissions from permissions@acm.org.

NetAI '19, August 23, 2019, Beijing, China

(C) 2019 Association for Computing Machinery.

ACM ISBN 978-1-4503-6872-8/19/08 . .\$15.00

https://doi.org/10.1145/3341216.3342212
ACM Reference Format:

Vidit Saxena, Joakim Jaldén, Joseph E. Gonzalez, Mats Bengtsson, Hugo Tullberg, and Ion Stoica. 2019. Contextual Multi-Armed Bandits for Link Adaptation in Cellular Networks. In NetAI '19: ACM SIGCOMM 2019 Workshop on Network Meets AI \& ML, August 23, 2019, Beijing, China. ACM, New York, NY, USA, 6 pages. https://doi.org/10.1145/3341216.3342212

\section{INTRODUCTION}

Wireless communication links operate within a complex timevarying environment. The quality of a wireless channel, in terms of its information-carrying capacity, depends on the attenuation and the phase rotation of the signal during its transmission over the air. The magnitude of signal degradation depends on several factors, including the carrier frequency, the transmission distance, the electromagnetic interactions at the transmitter, the receiver, and any intervening objects - some of which are typically in motion [15]. Therefore, to efficiently use the wireless channel, a wireless communication system needs to quickly navigate the complex link configuration space. Link adaptation refers to the problem of selecting the optimal transmission parameters in response to periodically observed, time-varying, wireless channel states. Given the widespread adoption of wireless communication, it should come as no surprise that link adaptation is an area of active research in cellular networks [3,9] as well as wireless local area networks [12].

A typical link adaptation scheme is composed of two control loops: an inner loop, and an outer loop, respectively. With the inner loop [12], the adaptation takes place over short time intervals, called transmission time intervals (TTIs). A wireless link estimates the instantaneous channel response by periodically transmitting pilot signals to the receiver in the current TTI, and using these estimates to set the transmission parameters for the next TTIs. Unfortunately, inner loop link adaptation is not always accurate. Instead, it is negatively affected by inaccurate link performance models and the delay and quantization of channel state feedback, which is done to reduce the communication overhead. Therefore, to improve the link adaptation performance, wireless systems employ an additional outer loop link adaptation (OLLA) that attempts to compensate for the shortcomings of the inner loop by observing the link behavior over a longer time scale, i.e., over several TTIs [20].

State-of-the-art OLLA techniques work by incrementally adjusting the link parameters to meet a configurable transmission failure rate. An optimally defined transmission failure rate, also known 
as the target error rate, selects transmission parameters that are neither too conservative (which would lead to an under-utilization of the channel), nor too aggressive (which would risk lowering the link throughput through frequent failures). However, OLLA suffers from two significant shortcomings: First, the target error rate depends on the long-term channel statistics in a mathematically intractable manner [17]. Second, even where the target error rate can be estimated accurately, OLLA may suffer from slow convergence and ultimately degrade the average link throughput [4].

In this paper, we propose a novel contextual multi-armed bandit (MAB) approach for link adaptation, where the context is provided by the periodically observed wireless channel state coupled with side information about the link. For a given context, the bandit arms model the success probability for each of the available transmission parameters. Subsequently, in each transmission instance, the arm that maximizes the expected link throughput is used for transmission. Based on the realized transmission success or failure event for the selected arm, the MAB is updated iteratively through online gradient updates. We show analytically that this scheme is converges to the true conditional success probabilities for each transmission parameter.

In contrast to OLLA, our approach does away with the need for finding a target error rate. Instead, our approach directly selects the throughput-maximizing parameter in each TTI. Further, our approach incorporates additional side information commonly available for wireless links, such as the link Doppler and the average strength of the channel. Finally, the contextual MAB used in our approach can exploit information from multiple, parallel, links to speed up the learning performance. Through numerical results from simulated experiments, we demonstrate that our approach converges faster than OLLA and improves the steady-state link throughput by up to $15 \%$ when the channel state reports are available in each TTI. When the channel reporting is done every 5 TTIs to reduce the signaling overhead, our approach increases the steady-state link throughput by up to $25 \%$ compared to OLLA.

\subsection{Related Work}

Several techniques have been proposed to improve the performance of OLLA. An enhanced OLLA scheme with dynamically adaptive step-sizes was proposed in [3] based on extensive theoretical analysis. This scheme was demonstrated to achieve up to $15 \%$ throughput gains compared to OLLA. Further to improve OLLA's convergence rate, [9] proposed learning the optimal initial OLLA offset from historical information of large-activity connections in practical cellular networks. In [8], sequential hypothesis testing was proposed to optimally set OLLA parameters and improve its convergence rate. The proposed solutions to improve OLLA performance critically depend on the knowledge of the target error rate. However in [17], it was shown that the target error rate is a function of the a priori unknown channel statistics, and a mathematical approximation was derived under several simplifying assumptions.

Learning-based schemes for link adaptation have generated significant recent interest. For example a $M A B$ and kernel-based approaches for link adaptation were proposed in [13] and [21] respectively, where the output corresponds to a discrete set of parameter adjustments applied to the inner loop link adaptation. However, these learning approaches ultimately relies on defining the OLLA parameter offsets to achieve a target error rate, and consequently suffer from the design problems associated with OLLA. In the context of cognitive wireless networks, MABs have been proposed to select one out of several available (channel, rate) combinations during each TTI to maximize the throughput [6]. Recently, deep reinforcement learning has also been investigated for a similar problem [22]. The channel selection problem in cognitive radios motivate our proposed approach for link adaptation, however, none of the prior schemes incorporate contextual information or deal with channel reporting delays.

\section{WIRELESS LINK ADAPTATION}

\subsection{System Model}

A wireless receiver estimates the instantaneous channel state from known, deterministic, pilot signals transmitted periodically over the link. Subsequently, the receiver feeds back a quantized channel quality index (CQI), $q \in Q$, where the quantization trades off accuracy of the reported channel state with the signaling overhead. The transmitter uses this CQI for selecting the optimal transmission parameters in the next TTIs, indexed by a finite set of modulation and coding schemes (MCS). In each TTI indexed by $t=\{1, \ldots, T\}$, the choice of a MCS index, $m(t) \in \mathcal{M}$, dictates the number of data bits $D_{m(t)}$ that are packed into a transport block. The transmitter processes the transport block by first encoding the bits onto $N_{m(t)}$ bits through a prescribed error protection scheme for reliable reception. Subsequently, it modulates the encoded bits onto complex data symbols from a symbol alphabet for transmission over the air. For bandwidth and TTI duration $\Delta f$ and $\Delta t$ respectively, the data throughput of the transmission is given by

$$
R_{m(t)} \triangleq \frac{1}{\Delta t \Delta f} D_{m(t)} \quad[\text { bits } / \mathrm{s} / \mathrm{Hz}],
$$

i.e., the number of information bits transmitted in the TTI normalized over the used transmission resources. The transmitted signal encounters attenuation, phase rotation and additive noise before arriving at the receiver. The receiver applies channel compensation to the received signal and subsequently attempts to recover the transmitted information bits. If the transmitted bits are recovered accurately (verified using a cyclic redundancy check), the receiver reports back to the transmitter a positive acknowledgement (ACK), $c=1$, otherwise it reports a negative ACK, $c=0$.

The inner loop link adaptation technique approaches the problem of selecting the optimal MCS index in each TTI in the following manner: A table that maps the CQI to an effective signal-tonoise-and-interference ratio (SINR), $\gamma_{\text {eff }}$, for a simplistic channel model is computed offline and stored locally at the transmitter. Further, offline curves that map $\gamma_{\text {eff }}$ to the MCS error probabilities are also generated numerically and made available to the transmitter. Consequently for a CQI value $q(t)$ reported by the receiver, the transmitter estimates the transmission error probabilities, $P\{c(t)=0 \mid m, q(t)\} \forall m \in \mathcal{M}$, and the corresponding expected throughputs

$$
\begin{aligned}
& R_{m} \cdot P\{c(t)=1 \mid m, q(t)\} \\
\approx & R_{m} \cdot P\left\{c(t)=1 \mid m, \gamma_{\mathrm{eff}}(t)\right\} \forall m \in \mathcal{M} .
\end{aligned}
$$


Subsequently for transmission, inner loop link adaptation picks the MCS index that maximizes the predicted link throughput, $m(t)=$ $\arg \max _{m \in \mathcal{M}} R_{m} \cdot P\left\{c(t)=1 \mid m, \gamma_{\mathrm{eff}}(t)\right\}$. Since the choice of an MCS index describes the type of the channel code as well as the symbol alphabet, inner loop link adaptation is also known as adaptive modulation and coding. Several learning approaches that aim to maximize the offline lookup accuracy, and consequently increase the link throughput, have been proposed in the literature $[7,19]$.

\subsection{Outer Loop Link Adaptation}

The MCS error probabilities estimated during inner loop link adaptation are inaccurate on account of the quantized CQI values as well as systematic modeling errors in the offline lookup tables. Further, often there is a signaling delay between the CQI generation and eventual data transmission, which contributes errors due to the time-varying nature of the wireless channel. Outer loop link adaptation (OLLA) attempts to compensate for these inaccuracies by applying a dynamic offset, $\Delta_{\text {OLLA }}(t)$, to adjust the effective SINR

$$
\widehat{\gamma}_{\mathrm{eff}}(t)=\gamma_{\mathrm{eff}}(t)+\Delta_{\mathrm{OLLA}}(t)
$$

The adjusted effective SINR is fed to the offline lookup tables to obtain the (adjusted) transmission error probability for each MCS If the transmission is successful, $\Delta_{\text {OLLA }}(t)$ is stepped up by a fixed amount to reflect better SINR conditions than predicted, otherwise, it is stepped down by another, fixed, amount, i.e.,

$$
\Delta_{\text {OLLA }}(t+1)=\left\{\begin{array}{ll}
\Delta_{\text {OLLA }}(t)+\Delta_{\text {up }} & , c(t)=1 \\
\Delta_{\text {OLLA }}(t)-\Delta_{\text {down }} & , c(t)=0
\end{array} .\right.
$$

By appropriately setting the step size ratio, OLLA can be made to converge to a desired target error rate, TER [3]

$$
\frac{\Delta_{\text {down }}}{\Delta_{\text {up }}}=\frac{\text { TER }}{1-\text { TER }} \text {. }
$$

The value of the TER is often set to 0.1 , which has been empirically verified to perform well for the most common link configurations [9]. Subsequently, OLLA selects the optimal MCS index in each TTI to maximize the expected throughput,

$$
m_{\text {OLLA }}(t)=\arg \max _{m \in \mathcal{M}} R_{m} \cdot P\left\{c(t)=1 \mid m, \widehat{\gamma}_{\mathrm{eff}}(t)\right\} .
$$

In [17], it was shown that the value of TER that maximizes the link throughput is not a single, fixed, value. Instead it depends on the channel statistics and the link configuration. Another shortcoming of OLLA is apparent from Eq. 6: The OLLA step sizes, and consequently the OLLA convergence rate, depend on the choice of target error rate as well.

\section{CONTEXTUAL MAB APPROACH}

To overcome the shortcomings of OLLA, we propose a novel online machine learning approach that directly models the mapping between an observed channel state and the transmission success probabilities for the available MCSs. Unlike OLLA, which relies on a heuristically defined target error rate for convergence, our approach compensates for model inaccuracies by iteratively learning from the observed success and failure events. Further, our approach uses a rich link state representation that can learn from multiple links in parallel, which leads to faster convergence.
We propose a contextual MAB for link adaptation, where the bandit arms maps a context vector containing the observed link state to the success probability for each available MCS. Further, we define the link adaptation policy, $\pi \in \Pi$, which selects the optimal MCS index for a given context vector. The goal of link adaptation is to find the policy

$$
\begin{aligned}
\pi^{*} & =\underset{\pi \in \Pi}{\arg \max _{\pi} \mathcal{W}_{\pi}(T),} \\
\text { where } \mathcal{W}_{\pi}(T) & =\frac{1}{T} \sum_{t=1}^{T} R_{m_{\pi}(t)} c(t)
\end{aligned}
$$

is the realized average link throughput. Denoting by $n_{\pi}^{(m, s)}$ the number of times that MCS index $m$ is picked for channel state $s$, Eq. 8 can be approximated using Wald's lemma,

$$
\begin{aligned}
\mathcal{W}_{\pi}(T) & \approx \sum_{m, s} E\left[n_{\pi}^{(m, s)}\right] R_{m} E[c \mid m, s] \\
& =\sum_{m, s} E\left[n_{\pi}^{(m, s)}\right] R_{m} P\{c=1 \mid m, s\} .
\end{aligned}
$$

Based on this formulation, the problem of finding an optimal link adaptation scheme $\pi$ can be stated as the equivalent problem of designing a policy that solves

$$
\arg \max _{\pi \in \Pi} \sum_{m, s} E\left[n_{\pi}^{(m, s)}\right] R_{m} P\{c=1 \mid m, s\},
$$

To solve this problem, the learning agent needs to learn the a priori unknown probabilities, $P\{c=1 \mid m, s\}$, from bandit feedback for the previously selected MCS values. A non-contextual version of problem in Eq. 10 has been explored in [6] in the context of cognitive wireless networks. For the context-dependent link adaptation problem discussed here, we develop a contextual MAB formulation of that learns the transmission success probability for each available MCS.

In addition to the reported CQI values in Eq. 2, cellular network have access to additional side information about the link, which can be exploited to improve the link adaptation performance. Consequently, we augment the link context vector by adding two scalar variables: The long-term average link SINR $\gamma_{\text {avg }}$, and the relative speed of the receiver with respect to the transmitter $v$. The motivation for adding $\gamma_{\text {avg }}$ stems from the fact that in case of OLLA, access to this variable has been shown to improve the link performance [17]. Further, the CQI reporting is often delayed, which leads to inaccurate predictions of the transmission success probabilities depending on the relative speed at which the receiver is movinh. Thie problem can be mitigated through the introduction of the relative receiver speed $v$ to the link context. Consequently, we define the three-dimensional link context vector

$$
s(t):=\left[q(t), \gamma_{\mathrm{avg}}, v\right] .
$$

Efficient learning schemes, which sequentially explore the action space and exploit it to maximize a cumulative stochastic payoff, have been developed for contextual MABs where the payoff can be assumed to be a stationary linear function of the context vector [ 5 , 14]. For the link adaptation problem considered here, the mapping from an observed context vector $s(t)$ to the transmission success probabilities is a nonlinear function due to the non-linear channel coding and modulation operations. Therefore, we use an artificial 
neural network (ANN) to model the the arms of the contextual $M A B$, where the input the network is provided by the link context vector $s(t)$, and the output of the network is the predicted success probability for each MCS. In the next section, we show that by minimizing the cross entropy loss between the ANN output and the binary transmission success events, $c(t)$, the ANN learns the maximum likelihood parameters for the true transmission success probabilities in Eq. 10.

\subsection{Artificial Neural Network Model}

We assume that the conditional transmission success probability $P\{c=1 \mid m, s)$ for each MCS index $m \in \mathcal{M}$ belongs to a parameterized family of functions $g_{\widehat{\theta}}(s, m)$ of the context vector $s$, where $\widehat{\theta}$ are some learnable model parameters, i.e., we assume that there exist some $\theta$ for which $P\{c=1 \mid m, s\}=g_{\theta}(s, m)$. We will explicitly use an ANN to parameterize $g_{\widehat{\theta}}(s, m)$, where $s$ is the input to the ANN, where $\widehat{\theta}$ are the ANN weights, and where $g_{\widehat{\theta}}(s, m)$ is the $m^{\text {th }}$ output, normalized between [0,1] by a sigmoid activation function at the output layer. We will for convenience write $P\{c=1 \mid m, s ; \widehat{\theta}\}=g_{\widehat{\theta}}(s, m)$ to stress the fact that the ANN is used to express a family of $m \in \mathcal{M}$ parameterized transmission success probabilities. The ANN parameters are trained as follows: For the context vector $s_{l}(t)$ in each TTI $t \in\{1, \ldots, T\}$ and links $l \in \mathcal{L}$, an optimal MCS $m_{l}(t)$ is selected in order to maximize Eq. 2 , and the transmission success event $c_{l}(t)$ is observed. Subsequently, the 3-tuples $\left(s_{l}(t), m_{l}(t), c_{l}(t)\right) \forall l \in \mathcal{L}$ are stored in an experience buffer for training. In each training iteration, a mini-batch of the training samples is selected randomly from the experience buffer and used to update the ANN parameters through stochastic gradient descent. For an experience buffer containing $K=|\mathcal{L}|$ training samples in each TTI, this approach is used to stochastically minimize the cross entropy loss

$$
C E(\widehat{\theta})=\frac{1}{K} \sum_{k=1}^{K} c_{k} \ln \left(g_{\widehat{\theta}}\left(s_{k}, m_{k}\right)\right)+\left(1-c_{k}\right) \ln \left(1-g_{\widehat{\theta}}\left(s_{k}, m_{k}\right)\right),
$$

where $g_{\widehat{\theta}}\left(s_{k}, m_{k}\right)=P\left(c_{k}=1 \mid s_{k}, m_{k} ; \widehat{\theta}\right)$ is the $m_{k}^{\text {th }}$ element of the ANN output for the input vector $s_{k}$.

Proposition 1. Minimizing the cost function in Eq.12 is equivalent to finding $\theta$ such that $P\{c=1 \mid m, s\}=g_{\theta}(s, m)$.

Proof. We can collect the log terms in Eq. 12 to obtain

$$
\begin{aligned}
C E(\widehat{\theta}) & =\frac{1}{K} \sum_{k=1}^{K} \ln \left(g_{\widehat{\theta}}\left(s_{k}, m_{k}\right)\right)^{c_{k}}\left(1-g_{\widehat{\theta}}\left(s_{k}, m_{k}\right)\right)^{\left(1-c_{k}\right)} \\
& =\frac{1}{K} \sum_{k=1}^{K} \ln P\left(c_{k} \mid m_{k}, s_{k} ; \widehat{\theta}\right), \\
& =\frac{1}{K} \sum_{m \in \mathcal{M}} \sum_{i=1}^{n_{m}} \ln P\left(c_{i} \mid m_{i}, s_{i} ; \widehat{\theta}\right),
\end{aligned}
$$

where $n_{m}$ denotes the number of training samples for MCS index $m$. The optimal ANN parameters obtained by minimizing the cross entropy loss are then given by

$$
\begin{aligned}
\widehat{\theta}^{*} & =\arg \min _{\widehat{\theta}} \frac{1}{K} \sum_{m \in \mathcal{M}} \sum_{i=1}^{n_{m}} \ln P\left(c_{i} \mid m, s_{i} ; \widehat{\theta}\right) \\
& \approx \arg \min _{\widehat{\theta}} \sum_{m \in \mathcal{M}} E[\ln P(c \mid m, s ; \widehat{\theta})],
\end{aligned}
$$

where the expectation is taken over the observations distributed according to the true probability model, $P(c \mid m, s ; \theta)$, and the approximation accounts for the finite size of the mini-batches. We subtract and add the true conditional probabilities to get

$$
\begin{aligned}
& =\sum_{m \in \mathcal{M}} E[\ln P(c \mid m, s ; \widehat{\theta})-\ln P(c \mid m, s ; \theta)+\ln P(c \mid m, s ; \theta)] \\
& =\sum_{m \in \mathcal{M}} E\left[\ln \frac{P(c \mid m, s ; \widehat{\theta})}{P(c \mid m, s ; \theta)}\right]+\ln P(c \mid m, s ; \theta) \\
& =-\sum_{m \in \mathcal{M}} K L\{P(c \mid m, s ; \theta) ; P(c \mid m, s ; \widehat{\theta})\}+E[\ln P(c \mid m, s ; \theta)],
\end{aligned}
$$

where $K L($.$) denotes the non-negative Kullback-Leibler (KL) diver-$ gence between the true and estimated probability distributions. The first term in the expression above is minimized when the KL divergence approaches zero, which implies that the estimated probability distribution closely approximates the true distribution. The second expectation term is independent of the ANN parameters and does not contribute to minimizing the cost function.

The ANN model training, while allowing a richer channel state, has a higher computational complexity as compared to OLLA. However, modern cellular base stations feature powerful multi-core processors designed for highly parallelized real-time operation. Moreover, future cellular networks will have access to additional, co-located, edge computing resources [10]. In such networks, the ANN training can be implemented using spare processing cycles in order to improve the network performance.

\section{EXPERIMENTAL RESULTS}

We simulate several, independent, fourth-generation $(4 \mathrm{G})$ cellular links operating within a vehicular wireless channel model [1, 11]. The wireless channel is modeled as a time- and frequency-varying channel that is correlated in time according to the link's Doppler frequency, and correlated in frequency according to a multipath propagation environment model [15, Sec. 2.1]. We assume that the wireless channel is known perfectly at the receiver, and is used to generate periodic CQI reports using a common offline link model. The simulation environment is implemented using the industrystandard package, ITPP (accessed through its Python wrapper, pyitpp [18]). Environment instances are encapsulated within an OpenAI Gym interface and the learning is distributed over multiple computation nodes using the Ray package [16].

For each link, the optimal MCS in each TTI is selected according to the link adaptation approaches discussed earlier. The transport block is encoded using a Turbo encoder, and spread over the available time and frequency resources using orthogonal frequency division multiplexing (OFDM). Each TTI spans a grid of 12 subcarriers in frequency and 14 OFDM symbols in time. For each TTI, the average link throughput is obtained by calculating the mean number of bits transmitted successfully over the $L$ links. We simulate 
Table 1: Cellular Link Parameters

\begin{tabular}{ll}
\hline Parameter & Value \\
\hline Carrier frequency & $2 \mathrm{GHz}$ \\
Number of subcarriers & 12 \\
OFDM symbols in each TTI & 14 \\
Subcarrier spacing & $15 \mathrm{kHz}$ \\
TTI duration & $1 \mathrm{~ms}$ \\
Channel model & ITU Vehicular B [11] \\
Number of links & $L=256$ \\
Relative speeds & $\mathcal{N}(30,10) \mathrm{kmph}$ \\
Long-term average SINRs & $\mathcal{N}(20,5) \mathrm{dB}$ \\
OLLA step size scaling & 0.1 \\
\hline
\end{tabular}

Table 2: ANN Hyper-parameters

\begin{tabular}{ll}
\hline Parameter & Value \\
\hline ANN layout & Fully Connected \\
ANN layer sizes & {$[16,8,8,16]$} \\
Activation function & ReLU \\
Learning rate & 0.0005 \\
Mini-batch size & 16 \\
SGD iterations & 8 \\
\hline
\end{tabular}

$|\mathcal{M}|=16$ of the the channel code rates and modulation orders from the standardized set of MCSs for $4 \mathrm{G}$ networks [1]. The simulation statistics are collected over $2^{14}$ independent channel realizations for generating the results. A complete list of the simulation parameters is available in Table 1.

Next, we illustrate and discuss the performance of three link adaptation approaches:

- OLLA: At the start of transmission over the link, OLLA uses the offline lookup tables described above to select the optimal MCS. In subsequent TTIs, OLLA adjusts the effective SINR value to such as to meet the target error rate, as described in 2.2. Note that using OLLA, in conjunction to inner loop link adaptation, is expected to improve throughput performance, as it compensates any systematic differences between the transmission success probabilities predicted by offline tables and the realized probabilities. We use a target error rate of $10 \%$ that is commonly used in the literature $[2,13]$.

- Contextual MAB with pre-training (CMAB w pre-training): Our approach, as described in Sec. 3. This approach directly maps the observed channel state to the predicted transmission success probability for each MCS. However, a potential shortcoming of this approach is that if the learning starts from scratch at the beginning of every link transmission, several TTIs will be wasted until convergence. To mitigate this problem, we pre-train the ANN model using the offline lookup tables already available for inner loop link adaptation. Therefore, our approach starts at a similar level as OLLA, and further refines the probability estimates using stochastic gradient descent of the ANN parameters using the observed transmission success and failure events.

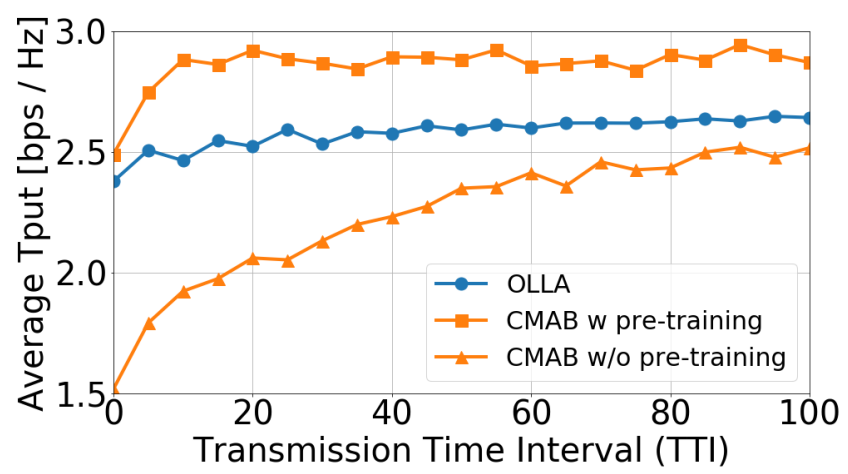

Figure 1: Average link throughput for OLLA, and link adaptation using contextual MAB with and without pre-training on the offline link model. The CQI is reported in every TTI.

- Contextual MAB without pre-training (CMAB w/o pre-training): Here we demonstrate the contextual MAB performance that does not pre-train on the offline link model. Instead, the MAB begins with a random initial state that is only trained online using the transmission feedback for the selected MCS value in each TTI as described in Sec. 3.

CQI reporting in every TTI: Frequent CQI reports allow accurate selection of the transmission parameters at the cost of a higher signaling overhead. Here we assume the maximum possible CQI reports, where a new CQI is generated in each TTI. In Fig. 1, we plot for the average link throughput (in bits per second per Hertz) across the simulated links for each of the discussed approaches. Contextual MAB approach with pre-training significantly outperforms OLLA on two accounts: faster convergence rate and steady-state link throughput. With pre-training, the contextual MAB approach converges much faster than OLLA, achieving its optimal link throughput in 10 TTIs. In contrast, with OLLA, the link throughout gradually increases for approximately 40 TTIs. This improved convergence for $\mathrm{MAB}$ is on account of pre-training as well as exploiting information from multiple parallel links. Further in the same plot, we observe that without pre-training, the contextual MAB approach performs poorly compared to OLLA.

In terms of the steady-state link throughput, contextual MAB with pre-training significantly outperforms OLLA, improving this performance metric by up to $15 \%$. The reason for these gains is that OLLA relies on the heuristically-defined target error of 0.1 . However, as discussed earlier, the target error rate depends on the channel statistics in a mathematically intractable manner, resulting in OLLA converging to a sub-optimal link throughput. On the other hand, our proposed contextual MAB approach mitigates this problem by automatically learning the optimal MCSs for the reported channel context without the need for any heuristically defined tuning parameters.

CQI reporting interval of 5 TTIs: Since CQI reports constitute a signaling overhead, it is desirable to reduce the reporting interval for communication efficiency. However since the channel varies with time, delayed CQI reports lead to inaccurate prediction of the link performance, and consequent degradation of the link 


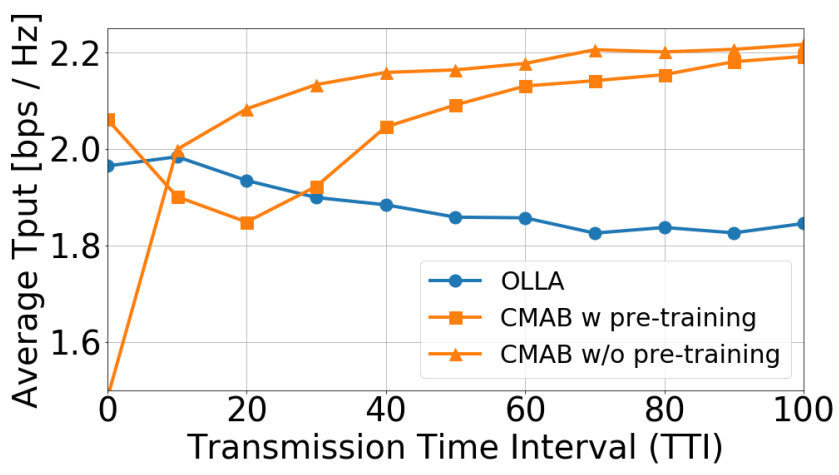

Figure 2: Average link throughput for OLLA and link adaptation using contextual MAB with and without pre-training on the offline link model. The CQI is reported every 5 TTIs.

throughput. In Fig. 2, we plot the OLLA and contextual MAB performance in case of a CQI reporting delay of 5 TTIs. We observe that OLLA actually leads to a degradation of the link throughput. The reason for this degradation is that OLLA applies SINR adjustments based on obsolete channel knowledge. Consequently, the OLLA adjustments end up introducing additional errors in the offline link model. Further with pre-training, the contextual MAB learns an inaccurate model of the link that does not incorporate the effect of CQI reporting delays. Subsequently, the gradient updates to the ANN model take several iterations to learn the true modeling parameters for the link. Therefore for the contextual MAB approach with pre-training, the link throughput degrades initially, before improving to a $25 \%$ higher steady-state throughput than that obtained with OLLA. In contrast, without pre-training, the contextual $\mathrm{MAB}$ approach converges faster to the steady-state link throughput. However, for this approach, the link throughput for the first few TTIs is poor compared to the competing approaches.

\section{CONCLUSIONS}

We have proposed a novel online learning approach to solve the problem of selecting the optimal MCS for link adaptation in cellular communication systems. We formulate this problem as an online stochastic policy optimization problem and solve it using a contextual MAB. Further within this approach, we use an ANN model to predict the transmission success probability for each MCS given a link context vector that contains the reported channel state and additional link side information. Finally, we apply our approach for selecting the optimal MCS that maximizes the link throughput and provide numerical results which demonstrate up to $25 \%$ increase in average link throughput compared to OLLA.

\section{ACKNOWLEDGEMENT}

This work was partially supported by the Wallenberg AI, Autonomous Systems and Software Program (WASP) funded by the Knut and Alice Wallenberg Foundation, and the European Research Council (ERC) under the European Union's Horizon 2020 research and innovation programme (grant agreement No 742648).

\section{REFERENCES}

[1] 3rd Generation Partnership Project. 2016. Evolved Universal Terrestrial Radio Access (E-UTRA); Physical layer procedures. Technical Report 36.213 v12.3.0.

[2] Kari Aho, Olli Alanen, and Jorma Kaikkonen. 2011. CQI reporting imperfections and their consequences in LTE networks. In Proceedings of ICN.

[3] Francisco Blanquez-Casado, Gerardo Gomez, Maria del Carmen Aguayo-Torres, and Jose Tomas Entrambasaguas. 2016. eOLLA: an enhanced outer loop link adaptation for cellular networks. EURASIP fournal on Wireless Communications and Networking 2016, 1 (16 Jan 2016), 20. https://doi.org/10.1186/s13638-016-0518-3

[4] V. Buenestado, J. M. Ruiz-Avilés, M. Toril, S. Luna-Ramírez, and A. Mendo. 2014. Analysis of Throughput Performance Statistics for Benchmarking LTE Networks. IEEE Communications Letters 18, 9 (Sept 2014), 1607-1610. https://doi.org/10. 1109/LCOMM.2014.2337876

[5] Wei Chu, Lihong Li, Lev Reyzin, and Robert Schapire. 2011. Contextual bandits with linear payoff functions. In Proceedings of the Fourteenth International Conference on Artificial Intelligence and Statistics. 208-214.

[6] R. Combes and A. Proutiere. 2015. Dynamic Rate and Channel Selection in Cognitive Radio Systems. IEEE Journal on Selected Areas in Communications 33, 5 (May 2015), 910-921. https://doi.org/10.1109/JSAC.2014.2361084

[7] R. C. Daniels, C. M. Caramanis, and R. W. Heath. 2010. Adaptation in Convolutionally Coded MIMO-OFDM Wireless Systems Through Supervised Learning and SNR Ordering. IEEE Transactions on Vehicular Technology 59, 1 (Jan 2010), 114-126.

[8] R. A. Delgado, K. Lau, R. Middleton, R. S. Karlsson, T. Wigren, and Y. Sun. 2017. Fast Convergence Outer Loop Link Adaptation with Infrequent Updates in Steady State. In 2017 IEEE 86th Vehicular Technology Conference (VTC-Fall). 1-5. https: //doi.org/10.1109/VTCFall.2017.8288232

[9] A. Durán, M. Toril, F. Ruiz, and A. Mendo. 2015. Self-Optimization Algorithm for Outer Loop Link Adaptation in LTE. IEEE Communications Letters 19, 11 (Nov 2015), 2005-2008. https://doi.org/10.1109/LCOMM.2015.2477084

[10] Yun Chao Hu, Milan Patel, Dario Sabella, Nurit Sprecher, and Valerie Young. 2015. Mobile edge computing - A key technology towards 5G. ETSI white paper 11, 11 (2015), 1-16.

[11] ITU-R. 1997. Guidelines for evaluation of radio transmission technologies for IMT-2000. Technical Report.

[12] T. L. Jensen, S. Kant, J. Wehinger, and B. H. Fleury. 2010. Fast Link Adaptation for MIMO OFDM. IEEE Transactions on Vehicular Technology 59, 8 (Oct 2010), 3766-3778. https://doi.org/10.1109/TVT.2010.2053727

[13] S. Katri Pulliyakode and S. Kalyani. 2017. Reinforcement learning techniques for Outer Loop Link Adaptation in 4G/5G systems. ArXiv e-prints (Aug. 2017). arXiv:stat.ML/1708.00994

[14] Lihong Li, Wei Chu, John Langford, and Robert E Schapire. 2010. A contextualbandit approach to personalized news article recommendation. In Proceedings of the 19th international conference on World wide web. ACM, 661-670.

[15] A.F. Molisch. 2010. Wireless Communications. Wiley.

[16] Philipp Moritz, Robert Nishihara, Stephanie Wang, Alexey Tumanov, Richard Liaw, Eric Liang, Melih Elibol, Zongheng Yang, William Paul, Michael I Jordan, et al. 2018. Ray: A distributed framework for emerging $\{\mathrm{AI}\}$ applications. In 13th $\{U S E N I X\}$ Symposium on Operating Systems Design and Implementation ( $\{O S D I\}$ 18). 561-577.

[17] S. Park, R. C. Daniels, and R. W. Heath. 2015. Optimizing the Target Error Rate for Link Adaptation. In 2015 IEEE Global Communications Conference (GLOBECOM). 1-6. https://doi.org/10.1109/GLOCOM.2015.7417770

[18] Vidit Saxena. 2016. py-itpp: Python library for signal processing and communications research. https://github.com/vidits-kth/py-itpp

[19] V. Saxena, J. Jaldén, M. Bengtsson, and H. Tullberg. 2018. Deep Learning for Frame Error Probability Prediction in BICM-OFDM Systems. In 2018 IEEE International Conference on Acoustics, Speech and Signal Processing (ICASSP). 6658-6662. https: //doi.org/10.1109/ICASSP.2018.8461864

[20] M. Shikh-Bahaei. 2007. Joint Optimization Of Transmission Rate and Outer-Loop SNR Target Adaptation Over Fading Channels. IEEE Transactions on Communications 55, 3 (March 2007), 398-403. https://doi.org/10.1109/TCOMM.2007.892441

[21] Sander Wahls and H Vincent Poor. 2013. An outer loop link adaptation for BICMOFDM that learns. In 2013 IEEE 14th Workshop on Signal Processing Advances in Wireless Communications (SPAWC). IEEE, 719-723.

[22] Shangxing Wang, Hanpeng Liu, Pedro Henrique Gomes, and Bhaskar Krishnamachari. 2018. Deep reinforcement learning for dynamic multichannel access in wireless networks. IEEE Transactions on Cognitive Communications and Networking 4, 2 (2018), 257-265. 